Slavica

bruxellensia

\section{Slavica bruxellensia}

Revue polyphonique de littérature, culture et histoire

slaves

$11 \mid 2015$

Littérature et philosophie

\title{
Lubomír Doležel, Heterocosmica II. Fikční světy postmoderní české prózy
}

\section{Martin Kolár̆}

\section{OpenEdition}

\section{Journals}

Édition électronique

URL : http://journals.openedition.org/slavica/1729

DOI : 10.4000/slavica.1729

ISSN : 2034-6395

\section{Éditeur}

Université libre de Bruxelles - ULB

\section{Référence électronique}

Martin Kolář, «Lubomír Doležel, Heterocosmica II. Fikční světy postmoderní české prózy », Slavica bruxellensia [En ligne], 11 | 2015, mis en ligne le 15 février 2015, consulté le 22 septembre 2020. URL http://journals.openedition.org/slavica/1729; DOI : https://doi.org/10.4000/slavica.1729

Ce document a été généré automatiquement le 22 septembre 2020.

\section{(c) $(1) \&$}

Les contenus de Slavica bruxellensia sont mis à disposition selon les termes de la Licence Creative Commons Attribution - Pas d'Utilisation Commerciale - Pas de Modification 3.0 France. 


\title{
Lubomír Doležel, Heterocosmica II. Fikční světy postmoderní české prózy
}

\author{
Martin Kolář
}

\section{RÉFÉRENCE}

Lubomír Doležel, Heterocosmica II. Fikční světy postmoderní české prózy (Heterocosmica II. Les mondes fictifs de la prose postmoderne tchèque), Karolinum, Prague, 2014, 192 p. 
1 «En ce qui me concerne, l'histoire de la littérature est avant tout l'histoire des styles. La littérature est belle et fascinante pour moi justement à cause de sa variété. En cela la littérature est plus riche que la vie car la vie consiste en histoires du réel tandis que la littérature consiste en histoires du possible. Et, comme a dit un philosophe, la sphère du possible est plus large que la sphère du réel. ${ }^{1}$

Lubomír Doležel (né en 1922) appartient aux plus influents des chercheurs tchèques en théorie littéraire ${ }^{2}$. Il est professeur émérite de l'Université de Toronto où il a enseigné entre 1968-2002. A partir de 1965, il a travaillé à l'Université Ann Arbor du Michigan où il a dirigé la collection «Prague Studies in

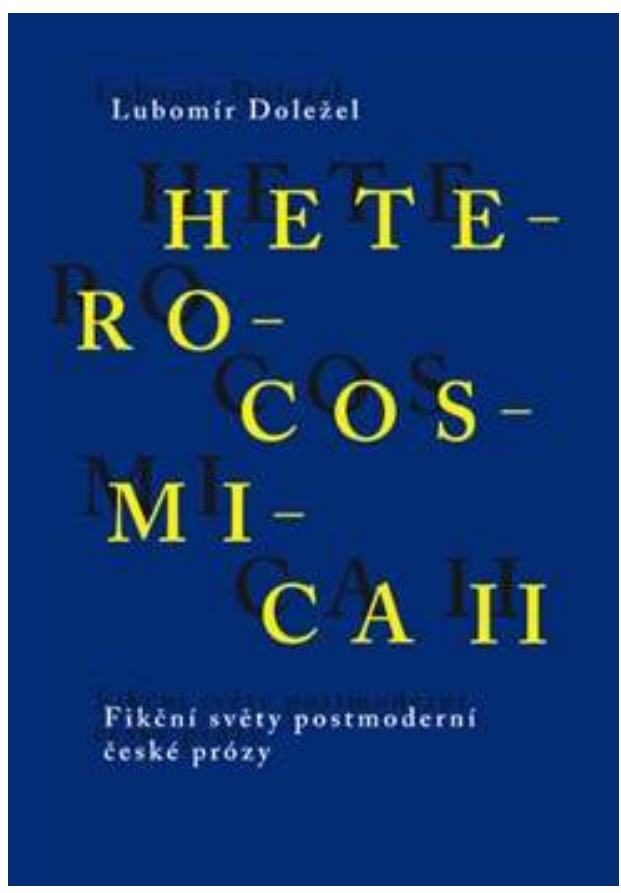
Mathematical Linguistics ».

3 Doležel appartient, ensemble Thomas G. Pavel, Marie-Laure Ryan et Umberto Eco, aux fondateurs de la théorie des mondes fictifs. Le concept des «mondes fictifs » part de la théorie des mondes possibles, développée par Gottfried Wilhelm Leibniz et actualisée dans la sémantique de la logique intuitionniste de Saul Kripke ${ }^{3}$. Les cadres de Kripke sont désignés dans différents types de logiques par le terme "états » ou " mondes possibles ». La pensée de Doležel trouve ses origines également dans la logique aléthique modale et se base sur la tradition du Cercle linguistique de Prague (Doležel est linguiste de formation) ainsi que sur les travaux de Jan Mukařovský et Felix Vodička.

4 Son nouveau livre est orienté selon deux axes. Le premier introduit les travaux de chercheurs tchèques qui s'intéressent à la thématique des mondes fictifs. Dans le domaine de la théorie littéraire tchèque, cette problématique est dominante et exerce une influence conséquente depuis les années 1990, grâce justement aux travaux de Doležel. Ainsi, la connaissance de son œuvre semble essentielle pour tous ceux qui s'intéressent à l'évolution de la théorie littéraire tchèque après 1989.

5 Parmi ceux qui ont été influencé par lui (ou ont réagi à ses travaux), nous pouvons mentionner Bohumil Fořt, Miloslava Slavíčková, Alice Jedličková, Petr KoŤátko, Jan Tlustý, Ondřej Sládek, Jiří Koten, Radomír D. Kokeš, Aleš Merenus, Zoltán Rédey, Vladimír Trpka, Michal Fránek, Dalibor Tureček.

6 Le deuxième axe suit et analyse une sélection d'œuvres considérées par l'auteur comme représentant la littérature postmoderne tchèque en faisant référence à la théorie, à la méthodologie et à la terminologie du premier volume, Heterocosmica. Fiction and Possible Worlds.

7 Si, dans ce premier volume, Doležel concentrait exclusivement son attention sur Směšné lásky (Risibles amours) et Kniha smichu a zapomneni (Livre du rire et de l'oubli) de Milan Kundera ainsi que sur Ostře sledované vlaky (Trains étroitement surveillés) de Bohumil Hrabal, dans le présent opus, il élargit son attention à d'autres auteurs tchèques, 
notamment Egon Hostovský, Karel Čapek, Karel Michal, Petr KoŤátko, Michal Ajvaz, Václav Řezáč, Karel Pecka, Daniela Hodrová, Jiř́i Kratochvil ou encore Jáchym Topol.

Déjà dans Heterocosmica I la théorie des mondes possibles permettait à Doležel de décrire l'ensemble de la structure aléthique des mondes fictifs. Dans Heterocosmica II, l'auteur met plutôt l'accent sur le point de départ de Leibniz, mis à l'écart par le positivisme, et il définit avec plus de précision la différence entre le monde possible et le monde fictif. L'auteur conçoit la théorie de point de vue de la pragmatique linguistique ${ }^{4}$. Il se base sur la théorie des actes illocutoires de John L. Austin, se concentrant particulièrement sur les performatifs. L'organisation de l'espace littéraire est strictement anti-mimétique et elle est dirigée par des raisons pragmatiques. On peut parler d'une sorte de "création du monde $»^{5}$. Il serait intéressant de comparer l'œuvre de Doležel avec la pensée de Nelson Goodman ou Arthur Danto ${ }^{6}$. Le point de départ structuraliste est clairement discernable, mais loin d'être institutionnalisé, Heterocosmica II l'oriente plutôt vers une fonction esthétique, telle qu'elle est conçue au sein de la tradition structuraliste tchèque. On pourrait également trouver une correspondance, même si elle n'est pas directement mentionnée, avec la définition de l'esthétique introduite par Monroe C. Beardsley ${ }^{7}$ qui définit l'œuvre artistique à la base de l'expérience ou du caractère esthétiques. Pour cette raison nous pouvons considérer ce livre comme une publication traditionnelle, du point de vue de la linguistique mais également de l'esthétique classique, telle qu'elle a été conçue par son fondateur, Alexander Gottlieb Baumgarten". Ainsi, en se basant sur le concept de la «fonction esthétique ", Dolezel défend l'idée de l'autonomie des mondes de la littérature et de l'art en général'.

\section{NOTES}

1. Doležel L., Heterocosmica II. Fikční světy postmoderní české prózy, Karolinum, Prague, 2014, p. 14.

2. Les travaux les plus importants de l'auteur sont : O stylu moderní české prózy (Du style dans la prose tchèque moderne), Academia, Prague, 1960 ; «Kafka's Fictional World" (Le monde fictionnel de Kafka), in: Canadian Review of Comparative Literature/Revue cannadiene de littérature comparé, n.11, 1984, pp.61-83 ; Occidental Poetics: Tradition and progres (La poétique occidentale : tradition et progrès), Lincoln University of Nebraska Press, 1990 ; Narativní zpưsoby včeské literatuře (Les moyens narratifs dans la littérature tchèque), Český spisovatel, Prague, 1993 ; Heterocosmica. Fiction and Possible Worlds (Heterocosmica. Mondes fictionnels et possibles), The John Hopkins University Press, Baltimore \& Londres, 1998 ; « Erotika a politika » (L'érotique et le politique), in: Slovo, strukturalismus, př́běh (Le mot, le structuralisme et l'histoire), sous la direction de Schneider J., Aluze, Olomouc, 2000, pp. 127-137; Fikce a historie v období postmoderny (Fictions et histoires à l'époque postmoderne), Academia, Prague, 2008; Studie z české literatury a poetiky (Etudes au sujet de la littérature tchèque et de la poétique), Torst, Prague, 2008 ; Possible Worlds of Fiction and History. The Postmodern Stage (Les mondes possibles de la fiction et de l'histoire. Le stade postmoderne), The John Hopkins University Press, Baltimore \& Londres, 2010. 
3. Kripke S. A., « Semantical Considerations on Modal Logic » (Considérations sémantiques sur la logique modale), in : Acta Philosophica Fennica, nº16, 1963, pp. 83-94.

4. Doležel admet qu'il est en désaccord avec la théorie de la fiction de John Searl et d'autres philosophes du langage. Doležel L., Heterocosmica II. op. cit., p. 12.

5. Le terme Worldmaking a été utilisé pour la première fois par Nelson Goodman en lien avec la construction de l'espace de l'œuvre artistique, Voir Goodman N., Ways of Worldmaking (Les manières de créer le monde), Hackett Publishing Company, Indiannapolis, 1978.

6. Danto A., " The Artworld » (Le monde-art), Philosophy Looks at the Arts (La philosophie regarde l'art), sous la direction de Margolis J., Temple University Press, Philadelphia, 1978, pp. 132-144.

7. „Work of arts (...) something produced with intention of giving it the capacity to satisfy the aesthetic interest." Beardsley M., C., An Aesthetic Definition of Art, in H. Curtler (ed.), What Is Art?, Haven Publishing Company, New York, 1983, p. 20.

8. Baumgarten A. G., Aesthetica, Kleyb, 1758.

9. Cette perspective mène Doležel à refuser l'esthétique de la réception de l'Ecole de Constance et à se s'autonomiser face au poststructuralisme. Voir Doležel L., Heterocosmica II, op. cit., p. 18.

\section{INDEX}

Index géographique : République tchèque, Tchécoslovaquie

Mots-clés : linguistique, littérature tchèque, structuralisme, théorie de la littérature

\section{AUTEURS}

\section{MARTIN KOLÁŘ}

Enseignant d'esthétique, Faculté d'Art et de Design, Université de Ústí nad Labem 